Textures and Microstructures, Vol. 33, pp. 337-341 Reprints available directly from the publisher Photocopying permitted by license only
(C) 1999 OPA (Overseas Publishers Association) N.V.

Published by license under the Gordon and Breach Science Publishers imprint. Printed in Malaysia.

\title{
OPTIMAL CALCULATION OF ODF WITH THE CANONICAL NORMAL DISTRIBUTION ON THE ROTATION GROUP
}

\author{
T.I. SAVYOLOVA*, E.A. DAVIDZHAN and T.M. IVANOVA \\ Moscow Engineering Physics Institute, Kashirskoe Shosse 31, \\ Moscow 113409, Russia
}

(Received 26 June 1997)

\begin{abstract}
Macroscopic physical properties of most polycrystalline materials are controlled by orientation distribution of their grains. The orientation distribution function (ODF) of a polycrystal is seldom if ever determined directly from an experiment. Usually experimental data are represented by a set of pole figures (PFs), these latter are some integral projections of the ODF. The main problem of quantitative texture analysis is to recover ODF from its corresponding PFs. With any set of PFs the solution of this problem is non-unique. That is why some assumptions about ODF structure are necessary. We consider ODF as superposition of the canonical normal distribution (CND) on the rotation group $S O(3)$.
\end{abstract}

Keywords: Orientation distribution function; Canonical normal distribution

\section{ROTATION PARAMETERS AND REPRESENTATION}

All kinds of rotations in the 3-dimensional space form the rotation group $S O(3)$. Let a unitary representation of the group be specified, i.e. unitary operators $T_{g}$ exist for any rotation $g \in S O(3)$ so that

$$
\begin{aligned}
T_{g_{1 g_{2}}} & =T_{g_{1}} T_{g_{2}}, \\
T_{e} & =I .
\end{aligned}
$$

If a rotation is defined by parameters $\left(\xi_{1}, \xi_{2}, \xi_{3}\right)$ (where $\vec{\xi}$ coincides with the rotation axis, and $|\vec{\xi}|=\delta$ is equal to the rotation angle), then we

\footnotetext{
* Corresponding author.
} 
get

$$
T(g) \equiv T\left(\xi_{1}, \xi_{2}, \xi_{3}\right)=\mathrm{e}^{\xi_{1} A_{1}+\xi_{2} A_{2}+\xi_{3} A_{3}},
$$

where $A_{1}, A_{2}, A_{3}$ are the operators of infinitesimal rotations around coordinate axes. These operators completely define the representation (Gelfand et al., 1958). In the basis of the eigen vectors of $A_{3}$ operator, $T_{g}$ takes the form of unitary matrix with generalised spherical functions $T_{l}^{m n}(g)$ as elements. If the rotation is defined by Eulerian angles $(\psi, \vartheta, \varphi)$, then we have (Vilenkin, 1991)

$$
T_{l}^{m n}(g)=\mathrm{e}^{-\mathrm{i} m \varphi-\mathrm{i} n \psi} P_{l}^{m n}(\cos \vartheta) .
$$

The parameters $\left(\xi_{1}, \xi_{2}, \xi_{3}\right)$ and $(\psi, \vartheta, \varphi)$ are related by the following equations:

$$
\left\{\begin{array}{l}
\xi_{1}=s \delta \sin (\vartheta / 2) \cos ((\psi-\varphi) / 2) / \sin (\delta / 2) \\
\xi_{2}=s \delta \sin (\vartheta / 2) \sin ((\psi-\varphi) / 2) / \sin (\delta / 2) \\
\xi_{3}=s \delta \cos (\vartheta / 2) \sin ((\psi+\varphi) / 2) / \sin (\delta / 2) \\
\cos (\delta / 2)=s \cos (\vartheta / 2) \cos ((\psi+\varphi) / 2) \\
s=\operatorname{sign}(\cos ((\psi+\varphi) / 2))
\end{array}\right.
$$

\section{DEFINITION}

Consider the canonical normal distribution (CND) on the group $S O(3)$. Let $\left\{T_{l}(g)\right\}$ be a set of irreducible representations of $S O(3)$. The distribution density $f(g)$ is said to be canonical normal if it admits representation of the following type (Savyolova, 1984; 1993):

$$
\int_{S O(3)} T_{l}(g) f(g) \mathrm{d} g=\mathrm{e}^{\alpha_{11}\left(A_{1}^{l}\right)^{2}+\alpha_{22}\left(A_{2}^{l}\right)^{2}+\alpha_{33}\left(A_{3}^{\prime}\right)^{2}},
$$

where $\alpha_{11}, \alpha_{22}, \alpha_{33}$ are non-negative constants and $\mathrm{d} g$ is the invariant measure on $S O(3)$,

$$
d g=\sin \vartheta d \vartheta d \psi d \varphi=\frac{\sin ^{2}(\delta / 2)}{(\delta / 2)^{2}} d \xi_{1} d \xi_{2} d \xi_{3} .
$$


In this case we have the formula

$$
\begin{aligned}
f(g) & =\sum_{l=0}^{\infty}(2 l+1) \operatorname{Sp}\left(\mathrm{e}^{\alpha_{11}\left(A_{1}^{l}\right)^{2}+\alpha_{22}\left(A_{2}^{l}\right)^{2}+\alpha_{33}\left(A_{3}^{l}\right)^{2}} \mathrm{e}^{\xi_{1} A_{1}^{l}+\xi_{2} A_{2}^{l}+\xi_{3} A_{3}^{l}}\right) \\
& =\sum_{l=0}^{\infty} \sum_{m=-l}^{l} \sum_{n=-l}^{l} C_{l}^{m n} T_{l}^{m n}(g) .
\end{aligned}
$$

\section{PROPERTIES OF THE CND}

1. The function $f(g)$ has the same values for the following 8 rotations:

$$
\begin{array}{ll}
\left(\xi_{1}, \xi_{2}, \xi_{3}\right) \text { or in Eulerian angles } & (\psi, \vartheta, \varphi), \\
\left(\xi_{1}, \xi_{2},-\xi_{3}\right) & (2 \pi-\varphi, \vartheta, 2 \pi-\psi), \\
\left(\xi_{1},-\xi_{2}, \xi_{3}\right) & (\varphi, \vartheta, \psi), \\
\left(\xi_{1},-\xi_{2},-\xi_{3}\right) & (2 \pi-\psi, \vartheta, 2 \pi-\varphi), \\
\left(-\xi_{1}, \xi_{2}, \xi_{3}\right) & (\pi-\psi, \vartheta, \pi-\varphi), \\
\left(-\xi_{1}, \xi_{2},-\xi_{3}\right) & (\pi+\varphi, \vartheta, \pi+\psi), \\
\left(-\xi_{1},-\xi_{2}, \xi_{3}\right) & (\pi-\varphi, \vartheta, \pi-\psi), \\
\left(-\xi_{1},-\xi_{2},-\xi_{3}\right) & (\pi+\psi, \vartheta, \pi+\varphi) .
\end{array}
$$

This property allows to reduce calculation 8 times.

2. Consider the dependence of CND on the parameters $\left(\alpha_{11}, \alpha_{22}, \alpha_{33}\right)$. Now we designate $f \equiv\left(\alpha_{11}, \alpha_{22}, \alpha_{33}, \xi_{1}, \xi_{2}, \xi_{3}\right)$. Any permutation of distribution parameters $\left(\alpha_{11}, \alpha_{22}, \alpha_{33}\right)$ causes respective permutation of rotation parameters $\left(\xi_{1}, \xi_{2}, \xi_{3}\right)$, i.e.

$$
\begin{aligned}
f\left(\alpha_{11}, \alpha_{22}, \alpha_{33}, \xi_{1}, \xi_{2}, \xi_{3}\right) & =f\left(\alpha_{22}, \alpha_{11}, \alpha_{33}, \xi_{2}, \xi_{1}, \xi_{3}\right) \\
& =f\left(\alpha_{22}, \alpha_{33}, \alpha_{11}, \xi_{2}, \xi_{3}, \xi_{1}\right)=\ldots
\end{aligned}
$$

For example the permutation $\alpha_{11} \leftrightarrow \alpha_{22}$ is equal to the substitution $\psi \rightarrow \pi / 2-\psi, \varphi \rightarrow 3 \pi / 2-\varphi$.

3. The CND $f(g)$ peaks at the rotation $g=e=(0,0,0)$ and the maximum does not depend on the order of parameters $\left(\alpha_{11}, \alpha_{22}, \alpha_{33}\right)$,

$$
f(0,0,0)=\sum_{l=0}^{\infty}(2 l+1) \operatorname{Sp} \Lambda_{l}
$$

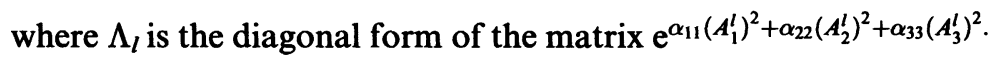




\section{APPROXIMATION OF CND}

Consider the distribution of the following type:

$$
\hat{f}(g)=1+\sum_{l=1}^{\infty}(2 l+1) \mathrm{e}^{-\varepsilon_{l}^{2} l(l+1)} \frac{\sin ((2 l+1) \hat{\delta} / 2)}{\sin (\hat{\delta} / 2)},
$$

where

$$
\hat{\delta}^{2}=\hat{\xi}_{1}^{2}+\hat{\xi}_{2}^{2}+\hat{\xi}_{3}^{2} \quad \text { with } \quad \hat{\xi}_{i}=\frac{\varepsilon_{l}}{\sqrt{\alpha_{i i}}} \xi_{i}
$$

and

$$
\varepsilon_{l}^{2}=\frac{1}{l(l+1)} \ln \frac{2 l+1}{\mathrm{Sp}\left(\Lambda_{l}\right)} .
$$

The distribution function $\hat{f}(g)$ satisfies the properties $1-3$. Numerical calculations reveal that $\hat{f}(g)$ approximates CND with sufficient accuracy. If the parameters $\left\{\alpha_{i i}\right\}$ are comparatively small $\left(\alpha_{i i}<\frac{1}{4}\right)$, then $\hat{f}(g)$ can be replaced by the formula

$$
\tilde{f}(g)=\frac{\sqrt{\pi}}{\sqrt{\alpha_{11} \alpha_{22} \alpha_{33}}} \exp \left\{-\left(\xi_{1}^{2} / \alpha_{11}+\xi_{2}^{2} / \alpha_{22}+\xi_{3}^{2} / \alpha_{33}\right)\right\} .
$$

The distribution $\tilde{f}(g)$ was proposed previously by Savyolova and Ivanova (1992). The numerical calculations of CND with different parameters are demonstrated in Savyolova and Davidzhan (1997).

\section{CONCLUSION}

The CND is the distribution of general kind for approximation ODF. The present work gives number of the CND properties and some approximation formulae.

\section{Acknowledgement}

The given work is executed pursuant to the program GRUNT from State Committee of Higher Education in Russia on fundamental researches in the field of geology. 


\section{References}

Gelfand, I.M., Minlos, R.A. and Shapiro, Z.J. (1958). Representation of Rotation Group and Loretz Group. Moscow, Fizmatgiz (in Russian).

Savyolova, T.I. (1984). Zavodskaya Laboratoriya, 50, 48-52 (in Russian).

Savyolova, T.I. (1993). Textures and Microstructures, 22, 17-27.

Savyolova, T.I. and Davidzhan, E.A. (1997). Zavodskaya Laboratoriya (in press, in Russian).

Savyolova, T.I. and Ivanova, T.M. (1992). Zavodskaya Laboratoriya, 52, 36-38 (in Russian).

Vilenkin, N.J. (1991). Special Functions and the Theory of Group Representation. Moscow, Nauka (in Russian). 